\title{
Vibration of a Rod-clutch System
}

\author{
L. Cveticanin \\ Faculty of Technical Sciences, 21000 Novi Sad, Trg D. Obradovica 6, Yugoslavia
}

The vibrations of a rod-clutch system are analyzed in this paper. The motion of the system is described with two second order nonlinear differential equations. The parameters of the clutch define the character of the rod motion. If the motion of the rod is chaotic and the clutch the rod is connected to the rod with certain properties the motion of the rod becomes periodic or the clutch represents an absorber of vibrations. A numerical example is given to prove the analytical results.

\section{INTRODUCTION}

The rotation of a rod is usually accompanied by torsional vibrations. A part of the rotational energy is transformed into energy in the form of torsional vibrations. These vibrations are unacceptable because they can perturb the accuracy of the some processes. Many investigations have been done to devise methods for avoiding or reducing such vibrations. Some absorbers with certain rigidity and moment of inertia are usually applied. ${ }^{1-4}$ The aim of this paper is to show that a clutch which is mounted on a rod is also a type of absorber.

The partial differential equation for the torsional motion of a rod with damping and an external torque is

I.e.

$$
\rho I_{0} \frac{\partial^{2} \varphi}{\partial t^{2}}=G I_{0} \frac{\partial^{2} \varphi}{\partial x^{2}}+M+K_{1} \varphi-K_{3} \varphi^{3}-b \frac{\partial \varphi}{\partial t}
$$

$$
\frac{\partial^{2} \varphi}{\partial t^{2}}=c^{2} \frac{\partial^{2} \varphi}{\partial x^{2}}+\frac{M}{\rho I_{0}}+c_{1}^{*} \varphi-c_{3}^{*} \varphi^{3}-\beta \frac{\partial \varphi}{\partial t}
$$

Where $G I_{0}$ is the torsional rigidity of the $\operatorname{rod}, \rho$ is the specific mass of the rod, $I_{0}$ is the polar moment of inertia, $M$ is the torque, $k_{1}$ and $k_{3}$ are coefficients of rigidity, $b$ is the damping coefficient, $\varphi$ is the angle of torsion, $t$ is time and $x$ is the position along the rod,

$$
\begin{gathered}
\varphi \equiv \varphi(x, t), \quad M \equiv M(x, t), \quad c^{2}=\frac{G}{\rho}, \\
c_{1}^{*}=\frac{k_{1}}{\rho I_{0}}, \quad c_{3}^{*}=\frac{k_{3}}{\rho I_{0}}, \quad \beta=\frac{b}{\rho I_{0}} .
\end{gathered}
$$

The boundary conditions are

$$
\varphi(0, t)=0, \quad \frac{\partial \varphi(l, t)}{\partial x}=0
$$

where $l$ is the length of the rod. It is assumed that the elastic properties of the rod are non-linear. This model corresponds to most of the aircraft materials. ${ }^{5}$ The torque $M$ is distributed along the rod and so are the effects of the non-linearity and damping. Distributing the excitation torque in series we obtain

$$
\frac{M}{\rho I_{0}}=\sum_{k=1}^{\infty} M_{k}(t) \sin \frac{k \pi}{l} x
$$

Let us assume a solution of the partial differential equation in the form

$$
\varphi(x m t)=\varphi_{1}(t) \sin \frac{\pi x}{2 l} .
$$

The solution (6) satisfies the boundary conditions (4). Substituting (6) into (2) and multiplying it with the weight function $\sin (\pi x / l)$ and integrating it along the length of the rod $l$ gives

$$
\ddot{\varphi}_{1}=-c^{2}\left(\frac{\pi}{2 l}\right)^{2} \varphi_{1}+\frac{3 \pi}{2} M_{1}(t)+c_{1}^{*} \varphi_{1}-\frac{3}{5} c_{3}^{*} \varphi_{1}^{3}-\beta \dot{\varphi}_{1}
$$

where

$$
M_{1}(t)=\frac{2}{l} \int_{0}^{l} \sum_{k=1}^{\infty} M_{k}(t) \sin \frac{k \pi x}{l} \sin \frac{\pi x}{l} d x .
$$

Introducing

$a_{1}=c_{1}^{*}-c^{2}\left(\frac{\pi}{2 l}\right)^{2}>0, a_{3}=0.6 c_{3}^{*}, M(t)=\frac{3 \pi}{2} M_{1(t),(9)}$ gives

For

$$
\ddot{\varphi}_{1}-\alpha_{1} \varphi_{1}+\alpha_{3} \varphi_{1}^{3}+\beta \dot{\varphi}_{1}=p(t)
$$

$$
M(t)=M_{0} \cos \Omega t,
$$

the equation represents the Duffing equation which has been widely investigated. ${ }^{6,7}$ It is known that for some parameter values of this equation describes chaotic irregular motion of the system with one degree of freedom. The chaotic motion is unacceptable and methods for suppressing this chaos are developed in this paper. The influence of the parameters of the clutch mounted on the rod and its effect on eliminating chaotic motion are analyzed.

\section{MATHEMATICAL MODEL}

The model of the rod-clutch system is shown in Fig. 1. For the case when the rod is modelled as a single-mode system (10) and the clutch represents a dynamic conservative absorber with moment of inertia $J$ and rigidity $k$, the mathematical model of the system is

$$
\begin{gathered}
\ddot{\varphi}_{1}-\alpha_{1} \varphi_{1}+\alpha_{3} \varphi_{1}^{3}+m a_{a}\left(\varphi_{1}-\varphi_{3}\right)+\beta \dot{\varphi}_{1}=M(t), \\
\ddot{\varphi}_{3}+a_{a}\left(\varphi_{3}-\varphi_{1}\right)=0
\end{gathered}
$$

where the dimensionless parameters are

$$
a_{a}=\frac{k}{J}, \quad m=\frac{J}{\rho I_{0} l} .
$$

